\title{
A Subclass of Harmonic Univalent Functions with Varying Arguments Defined by Generalized Derivative Operator
}

\author{
E. A. Eljamal and M. Darus \\ School of Mathematical Sciences, Faculty of Science and Technology, \\ Universiti Kebangsaan Malaysia Selangor, 43600 Bangi, Malaysia \\ Correspondence should be addressed to M. Darus, maslina@ukm.my
}

Received 21 November 2011; Revised 1 January 2012; Accepted 16 January 2012

Academic Editor: Shelton Peiris

Copyright (C) 2012 E. A. Eljamal and M. Darus. This is an open access article distributed under the Creative Commons Attribution License, which permits unrestricted use, distribution, and reproduction in any medium, provided the original work is properly cited.

\begin{abstract}
Making use of the generalized derivative operator, we introduce a new class of complex valued harmonic functions which are orientation preserving and univalent in the open unit disc and are related to uniformly convex functions. We investigate the coefficient bounds, neighborhood, and extreme points for this generalized class of functions.
\end{abstract}

\section{Introduction}

A continuous complex-valued function $f=u+i v$ defined in a simply connected complex domain $D$ is said to be harmonic in $D$ if both $u$ and $v$ are real harmonic in $D$. Such functions can be expressed as

$$
f=h+\bar{g},
$$

where $h$ and $g$ are analytic in $D$. We call $h$ the analytic part and $g$ the coanalytic part of $f$. A necessary and sufficient condition for $f$ to be locally univalent and sense preserving in $D$ is that $|\bar{h}(z)|>|\bar{g}(z)|$ for all $z$ in $D$ (see [1]). Let $H$ be the class of functions of the form (1.1) that are harmonic univalent and sense preserving in the unit disk $U=\{z:|z|<1\}$ for which $f(0)=f_{z}(0)-1=0$. Then for $f=h+\bar{g} \in H$, we may express the analytic functions $h$ and $g$ as

$$
h(z)=z+\sum_{k=2}^{\infty} a_{k} z^{k}, \quad g(z)=z+\sum_{k=1}^{\infty} b_{k} z^{k}, \quad z \in U,\left|b_{1}\right|<1 .
$$


In 1984, Clunie and Sheil-Small [1] investigated the class $S_{H}$ as well as its geometric subclasses and obtained some coefficient bounds. Since then, there have been several related papers on $S_{H}$ and its subclasses. Now we will introduce a generalized derivative operator for $f=h+\bar{g}$ given by (1.2). For fixed positive natural $m$ and $\lambda_{2} \geq \lambda_{1} \geq 0$,

$$
D_{\lambda_{1}, \lambda_{2}}^{m, k} f(z)=D_{\lambda_{1}, \lambda_{2}}^{m, k} h(z)+\overline{D_{\lambda_{1}, \lambda_{2}}^{m, k} g(z)}, \quad z \in U,
$$

where

$$
\begin{gathered}
D_{\lambda_{1}, \lambda_{2}}^{m, k} h(z)=z+\sum_{k=2}^{\infty}\left(\frac{1+\left(\lambda_{1}+\lambda_{2}\right)(k-1)}{1+\lambda_{2}(k-1)}\right)^{m} a_{k} z^{k}, \\
D_{\lambda_{1}, \lambda_{2}}^{m, k} g(z)=\sum_{k=1}^{\infty}\left(\frac{1+\left(\lambda_{1}+\lambda_{2}\right)(k-1)}{1+\lambda_{2}(k-1)}\right)^{m} a_{k} z^{k} .
\end{gathered}
$$

We note that by specializing the parameters, especially when $\lambda_{1}=\lambda_{2}=0, D_{\lambda_{1}, \lambda_{2}}^{m, k}$ reduces to $D^{m}$ which is introduced by Salagean in [2].

Now we will introduce the following definition.

Definition 1.1. For $0 \leq \ell<1$, let $G_{H}\left(\ell, m, k, \lambda_{1}, \lambda_{2}\right)$ denote the subfamily of starlike harmonic functions $f \in H$ of the form (1.1) such that

$$
\operatorname{Re}\left\{\left(1+e^{i \psi}\right) \frac{z\left(D_{\lambda_{1}, \lambda_{2}}^{m, k} f(z)\right)^{\prime}}{z^{\prime}\left(D_{\lambda_{1}, \lambda_{2}}^{m, k} f(z)\right)}-e^{i \psi}\right\} \geq \ell
$$

for a suitable real $\psi$ and $z \in U$ where $\left(D_{\lambda_{1}, \lambda_{2}}^{m, k} f(z)\right)^{\prime}=(d / d \theta)\left(D_{\lambda_{1}, \lambda_{2}}^{m, k} f\left(r e^{i \theta}\right)\right),(d / d \theta)\left(z=r e^{i \theta}\right)$.

We also let $V_{H}\left(\ell, m k, \lambda_{1}, \lambda_{2}\right)=G_{H}\left(\ell, m k, \lambda_{1}, \lambda_{2}\right) \cap V_{H}$ where $V_{H}$ is the class of harmonic functions with varying arguments introduced by Jahangiri and Silverman [3] consisting of functions $f$ of the form (1.1) in $H$ for which there exists a real number $\phi$ such that

$$
\eta_{k}+(k-1) \phi \equiv \pi(\bmod 2 \pi), \quad \delta_{k}+(k-1) \phi \equiv 0 \quad(k \geq 2)
$$

where $\eta_{k}=\arg \left(a_{k}\right)$ and $\delta_{k}=\arg \left(b_{k}\right)$. The same class introduced in [4] with different differential operator.

In this paper, we obtain a sufficient coefficient condition for functions $f$ given by (1.2) to be in the class $G_{H}\left(\ell, m, k, \lambda_{1}, \lambda_{2}\right)$. It is shown that this coefficient condition is necessary also for functions belonging to the class $V_{H}\left(\ell, m, k, \lambda_{1}, \lambda_{2}\right)$. Further, extreme points for functions in $V_{H}\left(\ell, m, k, \lambda_{1}, \lambda_{2}\right)$ are also obtained.

\section{Main Result}

We begin deriving a sufficient coefficient condition for the functions belonging to the class $G_{H}\left(\ell, m, k, \lambda_{1}, \lambda_{2}\right)$. This result is contained in the following. 
Theorem 2.1. Let $f=h+\bar{g}$ given by (1.2). Furthermore, let

$$
\sum_{k=2}^{\infty}\left(\frac{2 k-1-\ell}{1-\ell}\left|a_{k}\right|+\frac{2 k+1+\ell}{1-\ell}\left|b_{k}\right|\right)\left(\frac{1+\left(\lambda_{1}+\lambda_{2}\right)(k-1)}{1+\lambda_{2}(k-1)}\right)^{m} \leq 1-\frac{3+\ell}{3-\ell} b_{1}
$$

where $0 \leq \ell<1$, then $f \in G_{H}\left(\ell, m, k, \lambda_{1}, \lambda_{2}\right)$.

Proof. We first show that if the inequality (2.1) holds for the coefficients of $f=h+\bar{g}$, then the required condition (1.5) is satisfied. Using (1.3) and (1.5), we can write

$$
\operatorname{Re}\left\{\left(1+e^{i \psi}\right) \frac{z\left(D_{\lambda_{1}, \lambda_{2}}^{m, k} h(z)\right)^{\prime}-\overline{z\left(D_{\lambda_{1}, \lambda_{2}}^{m, k} g(z)\right)^{\prime}}}{\left(D_{\lambda_{1}, \lambda_{2}}^{m, k} h(z)\right)+\overline{\left(D_{\lambda_{1}, \lambda_{2}}^{m, k} g(z)\right)}}-e^{i \psi}\right\}=\operatorname{Re} \frac{A(z)}{B(z)}
$$

where

$$
\begin{gathered}
A(z)=\left(1+e^{i \psi}\right)\left[z\left(D_{\lambda_{1}, \lambda_{2}}^{m, k} h(z)\right)^{\prime}-\overline{z\left(D_{\lambda_{1}, \lambda_{2}}^{m, k} g(z)\right)^{\prime}}\right]-e^{i \psi}\left(D_{\lambda_{1}, \lambda_{2}}^{m, k} h(z)\right)+\overline{\left(D_{\lambda_{1}, \lambda_{2}}^{m, k} g(z)\right)}, \\
B(z)=\left(D_{\lambda_{1}, \lambda_{2}}^{m, k} h(z)\right)+\overline{\left(D_{\lambda_{1}, \lambda_{2}}^{m, k} g(z)\right) .}
\end{gathered}
$$

In view of the simple assertion that $\operatorname{Re}(w) \geq \ell$ if and only if $|1-\ell+w| \geq|1+\ell-w|$, it is sufficies to show that

$$
|A(z)+(1-\ell) B(z)|-|A(z)-(1+\ell) B(z)| \geq 0 .
$$

Substituting for $A(z)$ and $B(z)$ the appropriate expressions in (2.4), we get

$$
\begin{aligned}
\mid A(z)+ & (1-\ell) B(z)|-| A(z)-(1+\ell) B(z) \mid \\
\geq & (2-\ell)|z|-\sum_{k=2}^{\infty}(2 k-\ell)\left(\frac{1+\left(\lambda_{1}+\lambda_{2}\right)(k-1)}{1+\lambda_{2}(k-1)}\right)^{m}\left|a_{k} \| z\right|^{k} \\
& -\sum_{k=2}^{\infty}(2 k+\ell)\left(\frac{1+\left(\lambda_{1}+\lambda_{2}\right)(k-1)}{1+\lambda_{2}(k-1)}\right)^{m}\left|b_{k} \| z\right|^{k} \\
& -\ell|z|-\sum_{k=2}^{\infty}(2 k-2-\ell)\left(\frac{1+\left(\lambda_{1}+\lambda_{2}\right)(k-1)}{1+\lambda_{2}(k-1)}\right)^{m}\left|a_{k} \| z\right|^{k}
\end{aligned}
$$




$$
\begin{aligned}
-\sum_{k=2}^{\infty}(2 k+2+\ell)\left(\frac{1+\left(\lambda_{1}+\lambda_{2}\right)(k-1)}{1+\lambda_{2}(k-1)}\right)^{m}\left|b_{k}\right||z|^{k} \\
\geq 2(1-\ell)|z|\left\{1-\frac{3+\ell}{1-\ell} b_{1}-\left(\sum _ { k = 2 } ^ { \infty } \left[\frac{2 k-1-\ell}{1-\ell}\left(\frac{1+\left(\lambda_{1}+\lambda_{2}\right)(k-1)}{1+\lambda_{2}(k-1)}\right)^{m}\left|a_{k}\right|\right.\right.\right. \\
\left.\left.\left.+\frac{2 k+1+\ell}{1-\ell}\left(\frac{1+\left(\lambda_{1}+\lambda_{2}\right)(k-1)}{1+\lambda_{2}(k-1)}\right)^{m}\left|b_{k}\right|\right]\right)\right\} \geq 0
\end{aligned}
$$

by virtue of the inequality (2.1). This implies that $f \in G_{H}\left(\ell, m, k, \lambda_{1}, \lambda_{2}\right)$.

Now we obtain the necessary and sufficient condition for function $f=h+\bar{g}$ be given with condition (1.6).

Theorem 2.2. Let $f=h+\bar{g}$ be given by (1.2). Then $f \in V_{H}\left(\ell, m, k, \lambda_{1}, \lambda_{2}\right)$ if and only if

$$
\sum_{k=2}^{\infty}\left(\frac{2 k-1-\ell}{1-\ell}\left|a_{k}\right|+\frac{2 k+1+\ell}{1-\ell}\left|b_{k}\right|\right)\left(\frac{1+\left(\lambda_{1}+\lambda_{2}\right)(k-1)}{1+\lambda_{2}(k-1)}\right)^{m} \leq 1-\frac{3+\ell}{3-\ell} b_{1},
$$

where $0 \leq \ell<1$.

Proof. Since $V_{H}\left(\ell, m, k, \lambda_{1}, \lambda_{2}\right) \subset G_{H}\left(\ell, m, k, \lambda_{1}, \lambda_{2}\right)$, we only need to prove the necessary part of the theorem. Assume that $f \in V_{H}\left(\ell, m, k, \lambda_{1}, \lambda_{2}\right)$, then by virtue of (1.3) to (1.5), we obtain

$$
\operatorname{Re}\left\{\left(1+e^{i \psi}\right)\left[\frac{z\left(D_{\lambda_{1}, \lambda_{2}}^{m, k} h(z)\right)^{\prime}-\overline{z\left(D_{\lambda_{1}, \lambda_{2}}^{m, k} g(z)\right)^{\prime}}}{\left(D_{\lambda_{1}, \lambda_{2}}^{m, k} h(z)\right)+\overline{\left(D_{\lambda_{1}, \lambda_{2}}^{m, k} g(z)\right)}}-\left(e^{i \psi}+\ell\right)\right]\right\} \geq 0 .
$$

The above inequality is equivalent to

$$
\begin{aligned}
\operatorname{Re}\left\{\left(z+\left(\sum_{k=2}^{\infty}\left[k\left(1+e^{i \psi}\right)-\ell-e^{i \psi}\right]\left(\frac{1+\left(\lambda_{1}+\lambda_{2}\right)(k-1)}{1+\lambda_{2}(k-1)}\right)^{m}\left|a_{k}\right| z^{k}\right.\right.\right. \\
\left.\left.\quad-\sum_{k=2}^{\infty}\left[k\left(1+e^{i \psi}\right)+\ell+e^{i \psi}\right]\left(\frac{1+\left(\lambda_{1}+\lambda_{2}\right)(k-1)}{1+\lambda_{2}(k-1)}\right)^{m}\left|b_{k}\right| \overline{z^{k}}\right)\right), \\
\left.\quad \times\left(z+\sum_{k=2}^{\infty}\left(\frac{1+\left(\lambda_{1}+\lambda_{2}\right)(k-1)}{1+\lambda_{2}(k-1)}\right)^{m}\left|a_{k}\right| z^{k}+\sum_{k=2}^{\infty}\left(\frac{1+\left(\lambda_{1}+\lambda_{2}\right)(k-1)}{1+\lambda_{2}(k-1)}\right)^{m}\left|b_{k}\right| \overline{z^{k}}\right)^{-1}\right\}
\end{aligned}
$$




$$
\begin{aligned}
=\operatorname{Re}\{ & \left((1-\ell)+\sum_{k=2}^{\infty}\left[k\left(1+e^{i \psi}\right)-\ell-e^{i \psi}\right]\left(\frac{1+\left(\lambda_{1}+\lambda_{2}\right)(k-1)}{1+\lambda_{2}(k-1)}\right)^{m}\left|a_{k}\right| z^{k-1}\right. \\
& -\frac{\bar{z}}{z} \sum_{k=2}^{\infty}\left[k\left(1+e^{i \psi}\right)+\ell+e^{i \psi}\right]\left(\frac{1+\left(\lambda_{1}+\lambda_{2}\right)(k-1)}{1+\lambda_{2}(k-1)}\right)^{m}\left|b_{k}\right| \bar{z}^{k-1} \\
& \left.\times\left(1+\sum_{k=2}^{\infty}\left(\frac{1+\left(\lambda_{1}+\lambda_{2}\right)(k-1)}{1+\lambda_{2}(k-1)}\right)^{m}\left|a_{k}\right| z^{k-1}+\frac{\bar{z}}{z} \sum_{k=2}^{\infty}\left(\frac{1+\left(\lambda_{1}+\lambda_{2}\right)(k-1)}{1+\lambda_{2}(k-1)}\right)^{m}\left|b_{k}\right| \overline{z^{k-1}}\right)^{-1}\right\}
\end{aligned}
$$

$\geq 0$.

This condition must hold for all values of $z$, such that $|z|=r<1$. Upon choosing $\phi$ according to (1.6) and noting that $\operatorname{Re}\left(-e^{i \psi}\right) \geq-\left|e^{i \psi}\right|=-1$, the above inequality reduces to

$$
\begin{aligned}
& \left((1-\ell)-\left(1-b_{1}\right)-\left[\sum_{k=2}^{\infty}(2 k-1-\ell)\left(\frac{1+\left(\lambda_{1}+\lambda_{2}\right)(k-1)}{1+\lambda_{2}(k-1)}\right)^{m}\left|a_{k}\right| r^{k-1}\right.\right. \\
& \left.\left.+(2 k+1+\ell)\left(\frac{1+\left(\lambda_{1}+\lambda_{2}\right)(k-1)}{1+\lambda_{2}(k-1)}\right)^{m}\left|b_{k}\right| r^{k-1}\right]\right) \\
& \times\left(1-\sum_{k=2}^{\infty}\left(\frac{1+\left(\lambda_{1}+\lambda_{2}\right)(k-1)}{1+\lambda_{2}(k-1)}\right)^{m}\left|a_{k}\right| r^{k-1}+\sum_{k=1}^{\infty}\left(\frac{1+\left(\lambda_{1}+\lambda_{2}\right)(k-1)}{1+\lambda_{2}(k-1)}\right)^{m}\left|b_{k}\right| r^{k-1}\right)^{-1} \geq 0 .
\end{aligned}
$$

If (2.6) does not hold, then the numerator in (2.9) is negative for $r$ sufficiently close to 1. Therefore, there exists a point $z_{0}=r_{0}$ in $(0,1)$ for which the quotient in $(2.9)$ is negative. This contradicts our assumption that $f \in V_{H}\left(\ell, m, k, \lambda_{1}, \lambda_{2}\right)$. We thus conclude that it is both necessary and sufficient that the coefficient bound inequality (2.6) holds true when $f \in V_{H}\left(\ell, m, k, \lambda_{1}, \lambda_{2}\right)$. This completes the proof of Theorem 2.2.

Theorem 2.3. The closed convex hull of $f \in V_{H}\left(\ell, m, k, \lambda_{1}, \lambda_{2}\right)$ (denoted by clco $V_{H}\left(\ell, m, k, \lambda_{1}, \lambda_{2}\right)$ ) is

$$
\left\{f(z)=z+\sum_{k=2}^{\infty}\left|a_{k}\right| z^{k}+\overline{\sum_{k=1}^{\infty}\left|b_{k}\right| z^{k}}: \sum_{k=2}^{\infty} k\left[\left|a_{k}\right|+\left|b_{k}\right|\right]<1-b_{1}\right\} .
$$

By setting $\lambda_{k}=(1-\ell) /\left((2 k-1-\ell)\left(\left(1+\left(\lambda_{1}+\lambda_{2}\right)(k-1)\right) /\left(1+\lambda_{2}(k-1)\right)\right)^{m}\right)$ and $\mu_{k}=(1+$ $\ell) /\left((2 k+1+\ell)\left(\left(1+\left(\lambda_{1}+\lambda_{2}\right)(k-1)\right) /\left(1+\lambda_{2}(k-1)\right)\right)^{m}\right)$, then for $b_{1}$ fixed, the extreme points for clco $V_{H}\left(\ell, m, k, \lambda_{1}, \lambda_{2}\right)$ are

$$
\left\{z+\lambda_{k} x z^{k}+\overline{b_{1} z}\right\} \cup\left\{z+\overline{b_{1} z+\mu_{k} x z^{k}}\right\}
$$

where $k \geq 2$ and $|x|=1-\left|b_{1}\right|$. 
Proof. Any function $f$ in clco $V_{H}\left(\ell, m, k, \lambda_{1}, \lambda_{2}\right)$ may be expressed as

$$
f(z)=z+\sum_{k=2}^{\infty}\left|a_{k}\right| e^{i \eta_{k}} z^{k}+\overline{b_{1} z}+\overline{\sum_{k=2}^{\infty}\left|b_{k}\right| e^{i \delta k} z^{k}},
$$

where the coefficients satisfy the inequality (2.1). Set $h_{1}(z)=z, g_{1}(z)=b_{1} z, h_{k}(z)=z+$ $\lambda_{k} e^{i \eta k} z^{k}, g_{k}(z)=b_{1} z+\mu_{k} e^{i \delta k} z^{k}$ for $k=2,3, \ldots$ Writing $\chi_{k}=\left|a_{k}\right| / \lambda_{k}, Y_{k}=\left|b_{k}\right| / \mu_{k}, k=2,3, \ldots$ and $X_{1}=1-\sum_{k=2}^{\infty} X_{k} ; Y_{1}=1-\sum_{k=2}^{\infty} Y_{k}$, we get

$$
f(z)=\sum_{k=1}^{\infty}\left(X_{k} h_{k}(z)+Y_{k} g_{k}(z)\right)
$$

In particular, setting

$$
\begin{gathered}
f_{1}(z)=z+\overline{b_{1} z} \\
f_{k(z)}=z+\lambda_{k} x z^{k}+\overline{b_{1} z}+\overline{\mu k y z^{k}}, \quad\left(k \geq 2,|x|+|y|=1-\left|b_{1}\right|\right) .
\end{gathered}
$$

We see that extreme points of clco $f \in V_{H}\left(\ell, m, k \lambda_{1}, \lambda_{2}\right) \subset\left\{f_{k(z)}\right\}$.

To see that $f_{1}(z)$ is not in extreme point, note that $f_{1}(z)$ may be written as

$$
f_{1}(z)=\frac{1}{2}\left\{f_{1}(z)+\lambda_{2}\left(1-\left|b_{1}\right|\right) z^{2}\right\}+\frac{1}{2}\left\{f_{1}(z)-\lambda_{2}\left(1-\left|b_{1}\right|\right) z^{2}\right\}
$$

a convex linear combination of functions in clco $V_{H}\left(\ell, m, k, \lambda_{1}, \lambda_{2}\right)$.

To see that $f_{m}$ is not an extreme point if both $|x| \neq 0$ and $|y| \neq 0$, we will show that it can then also be expressed as a convex linear combinations of functions in clco $V_{H}\left(\ell, m, k, \lambda_{1}, \lambda_{2}\right)$. Without loss of generality, assume $|x| \geq|y|$. Choose $\epsilon>0$ small enough so that $\epsilon>|x| /|y|$. Set $A=1+\epsilon$ and $B=1-|\epsilon x / y|$. We then see that both

$$
\begin{gathered}
t_{1}(z)=z+\lambda_{k} A x z^{k}+\overline{b_{1} z+\mu_{k} y B z^{k}}, \\
t_{2}(z)=z+\lambda_{k}(2-A) x z^{k}+\overline{b_{1} z+\mu_{k} y(2-B) z^{k}}
\end{gathered}
$$

are in clco $V_{H}\left(\ell, m, k, \lambda_{1}, \lambda_{2}\right)$ and that

$$
f_{k}(z)=\frac{1}{2}\left\{t_{1}(z)+t_{2}(z)\right\}
$$

The extremal coefficient bounds show that functions of the form (2.11) are the extreme points for clco $V_{H}\left(\ell, m, k, \lambda_{1}, \lambda_{2}\right)$, and so the proof is complete. 
Following Avici and Zlotkiewicz [5] and [6], we refer to the $\delta$-neighborhood of the functions $f(z)$ defined by (1.2) to be the set of functions $F$ for which

$$
N_{\delta}(f)=\left\{F(z)=z+\sum_{k=2}^{\infty} A_{k} z^{k}+\sum_{k=1}^{\infty} \overline{B_{k} z^{k}}, \sum_{k=2}^{\infty} k\left(\left|a_{k}-A_{k}\right|+\left|b_{k}-B_{k}\right|+\left|b_{1}-B_{1}\right|\right) \leq \delta\right\} .
$$

In our case, let us define the generalized $\delta$-neighborhood of $f$ to be the set

$$
\begin{aligned}
N_{\delta}(f)=\{ & F(z): \sum_{k=2}^{\infty}\left(\frac{1+\left(\lambda_{1}+\lambda_{2}\right)(k-1)}{1+\lambda_{2}(k-1)}\right)^{m}\left[(2 k-1-\ell)\left(\left|a_{k}-A_{k}\right|+(2 k+1+\ell)\right)\left|b_{k}-B_{k}\right|\right] \\
& \left.+(1-\ell)\left|b_{1}-B_{1}\right| \leq(1-\ell) \delta\right\} .
\end{aligned}
$$

Theorem 2.4. Let $f$ be given by (1.2). If $f$ satisfies the conditions

$$
\begin{aligned}
& \sum_{k=2}^{\infty} k(2 k-1-\ell)\left|a_{k}\right|\left(\frac{1+\left(\lambda_{1}+\lambda_{2}\right)(k-1)}{1+\lambda_{2}(k-1)}\right)^{m}+\sum_{k=1}^{\infty} k(2 k+1+\ell)\left|b_{k}\right|\left(\frac{1+\left(\lambda_{1}+\lambda_{2}\right)(k-1)}{1+\lambda_{2}(k-1)}\right)^{m} \\
& \quad \leq(1-\ell)
\end{aligned}
$$

where $0 \leq \ell<1$, and

$$
\delta=\frac{1-\ell}{3-\ell}\left(1-\frac{3+\ell}{1-\ell}\left|b_{1}\right|\right)
$$

then $N(f) \subset G_{H}\left(\ell, m, k, \lambda_{1}, \lambda_{2}\right)$.

Proof. Let $f$ satisfy (2.20) and $F(z)$ be given by

$$
F(z)=z+\overline{B_{1} z}+\sum_{k=2}^{\infty}\left(A_{k} z^{m}+\overline{B_{k} z^{k}}\right)
$$

which belong to $N(f)$. We obtain

$$
\begin{aligned}
(3+\ell)\left|B_{1}\right| & +\sum_{k=2}^{\infty}\left((2 k-1-\ell)\left|A_{k}\right|+(2 k+1+\ell)\left|B_{k}\right|\right)\left(\frac{1+\left(\lambda_{1}+\lambda_{2}\right)(k-1)}{1+\lambda_{2}(k-1)}\right)^{m} \\
\leq & (3+\ell)\left|B_{1}-b_{1}\right|+(3+\ell)\left|b_{1}\right| \\
& +\sum_{k=2}^{\infty}\left(\frac{1+\left(\lambda_{1}+\lambda_{2}\right)(k-1)}{1+\lambda_{2}(k-1)}\right)^{m}\left[(2 k-1-\ell)\left|A_{k}-a_{k}\right|+(2 k+1+\ell)\left|B_{k}-b_{k}\right|\right]
\end{aligned}
$$




$$
\begin{aligned}
& +\sum_{k=2}^{\infty}\left(\frac{1+\left(\lambda_{1}+\lambda_{2}\right)(k-1)}{1+\lambda_{2}(k-1)}\right)^{m}\left[(2 k-1-\ell)\left|a_{k}\right|+(2 k+1+\ell)\left|b_{k}\right|\right] \\
\leq & (1-\ell) \delta+(3+\ell)\left|b_{1}\right| \\
& +\frac{1}{3-\ell} \sum_{k=2}^{\infty} k\left(\frac{1+\left(\lambda_{1}+\lambda_{2}\right)(k-1)}{1+\lambda_{2}(k-1)}\right)^{m}\left((2 k-1-\ell)\left|a_{k}\right|+(2 k+1+\ell)\left|b_{k}\right|\right) \\
\leq & (1-\ell) \delta+(3+\ell)\left|b_{1}\right|+\frac{1}{3-\ell}\left[(1-\ell)-(3+\ell)\left|b_{1}\right|\right] \leq 1-\ell .
\end{aligned}
$$

Hence for $\delta=(1-\ell) /(3-\ell)\left(1-((3+\ell) /(1-\ell))\left|b_{1}\right|\right)$, we infer that $F(z) \in G_{H}\left(\ell, m, k, \lambda_{1}, \lambda_{2}\right)$ which concludes the proof of Theorem 2.4 .

\section{Acknowledgment}

The work here was supported by UKM-ST-06-FRGS0244-2010.

\section{References}

[1] J. Clunie and T. Sheil-Small, "Harmonic univalent functions," Annales Academiae Scientiarum Fennicae A, vol. 9, pp. 3-25, 1984.

[2] G. S. Salagean, "Subclasses of univalent functions," in Complex Analysis, vol. 1013 of Lecture Notes in Math, pp. 362-372, Springer, Berlin, Germany, 1983.

[3] J. M. Jahangiri and H. Silverman, "Harmonic univalent functions with varying arguments," International Journal of Applied Mathematics, vol. 8, no. 3, pp. 267-275, 2002.

[4] G. Murugusundaramoorthy, K. Vijaya, and R. K. Raina, "A subclass of harmonic functions with varying arguments defined by Dziok-Srivastava operator," Archivum Mathematicum, vol. 45, no. 1, pp. 37-46, 2009.

[5] Y. Avici and E. Zotkiewicz, "On harmonic univalent mappings," Annales Universitatis Mariae CurieSkłodowska A, vol. 44, pp. 1-7, 1990.

[6] S. Ruscheweyh, "Neighborhoods of univalent functions," Proceedings of the American Mathematical Society, vol. 81, no. 4, pp. 521-527, 1981. 


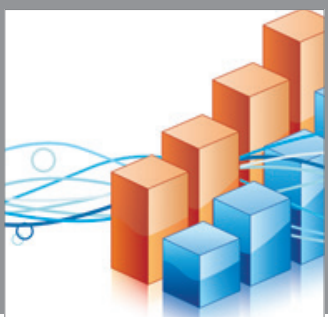

Advances in

Operations Research

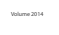

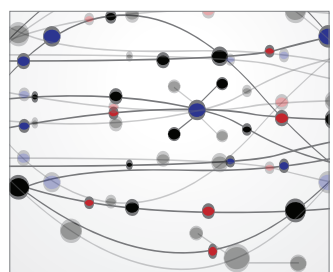

\section{The Scientific} World Journal
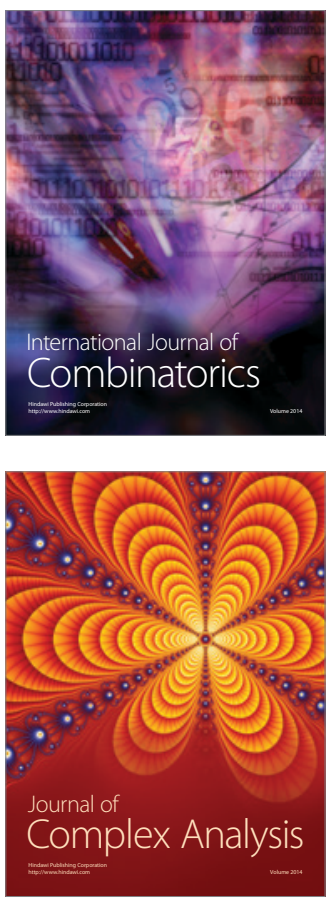

International Journal of

Mathematics and

Mathematical

Sciences
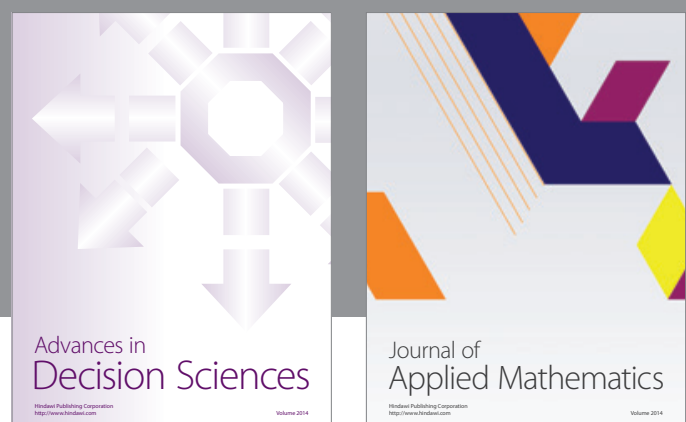

Journal of

Applied Mathematics
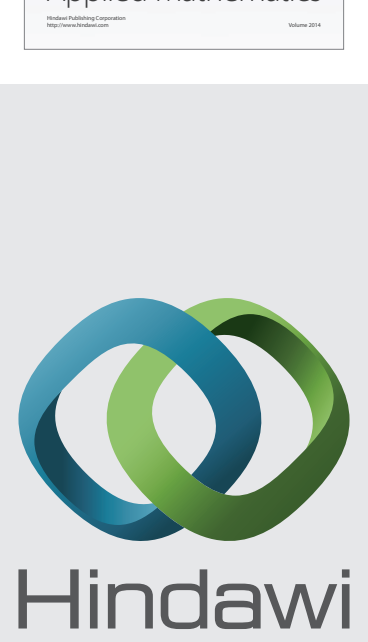

Submit your manuscripts at http://www.hindawi.com
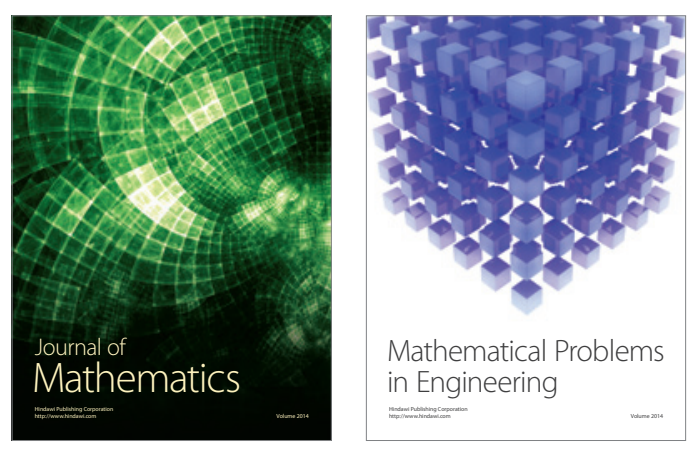

Mathematical Problems in Engineering
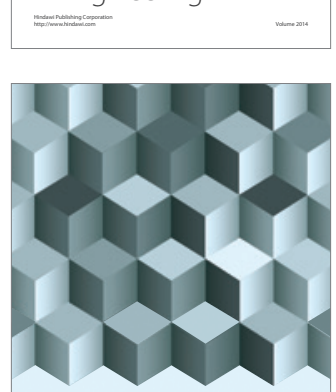

Journal of

Function Spaces
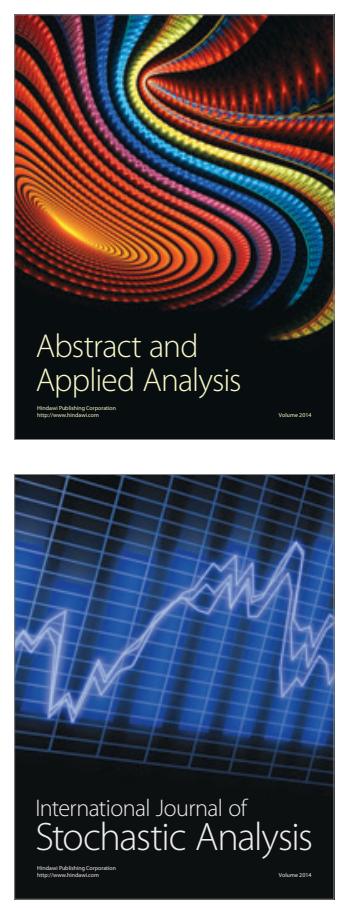

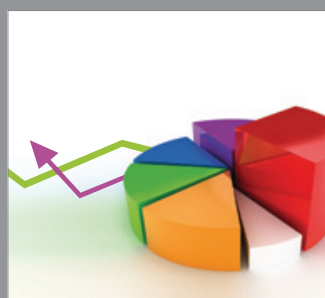

ournal of

Probability and Statistics

Promensencen
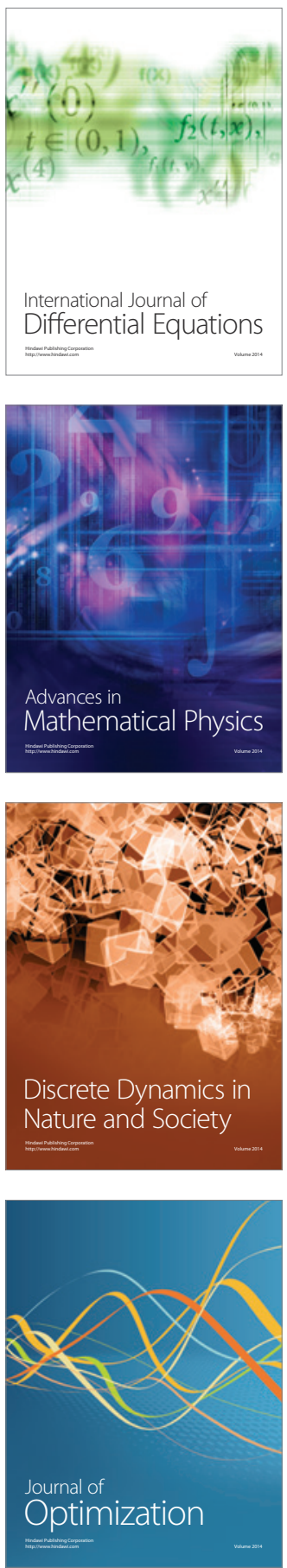